\title{
Equidad territorial en Medellín: espacio público, amenazas naturales y calidad del aire
}

\section{Territorial equity in Medellín: public space, natural hazards and air quality}

\author{
María Fernanda Cárdenas \\ PhD en Ingeniería. Escuela de Planeación Urbano Regional. Facultad de Arquitectura. Universidad Nacional de Colombia. Carrera 65 \#59a-110, \\ Medellín, Antioquia, Colombia, mfcarden@unal.edu.co, ORCID: 0000-0002-1804-6280 \\ Jhon Fredy Escobar \\ PhD en Administración. Facultad de Ciencias Adminsitrativas. Corporación Universitaria Remington. Calle 51 No. 51-27. Parque Berrio. Medellín, \\ Colombia, jfescob1@gmail.com, ORCID:0000-0002-6826-6222 \\ Katheryn Gutiérrez \\ Arquitecta. Cursando la maestría en Estudios Urbano Regionales. Escuela de Planeación Urbano Regional. Facultad de Arquitectura. Universidad \\ Nacional de Colombia. Carrera 65 \#59a-110, Medellín, Antioquia, Colombia, kgutierrezt@unal.edu.co
}

Recibido: 22 de noviembre 2019 || Aprobado: 14 de abril 2020

\section{Resumen}

En las ciudades latinoamericanas usualmente los mayores niveles socioeconómicos se asocian con mejores condiciones de vida, configurando segregación socioespacial. Este ejercicio evaluó la equidad territorial en la zona urbana de Medellín (Antioquia, Colombia), a escala de barrio, usando un índice de correlación espacial y gráficos de dispersión univariados y bivariados. Las variables usadas fueron espacio público, amenazas naturales y calidad del aire, que se relacionaron con el nivel socioeconómico prevalente de la población. Se encontró que en la ciudad de Medellín la distribución espacial de las variables evaluadas no se correlaciona con las condiciones socioeconómicas, tanto en aquellas deseables como espacio público, lo cual es producto de intervenciones recientes orientadas a equilibrar su distribución territorial; al igual que en las variables de polución y amenazas naturales que implican importantes retos de gestión y manejo territorial en toda la ciudad.

Palabras clave: Segregación socioespacial; Autocorrelación espacial; Valor de vivienda urbana; Índice I de Moran

\section{Abstract}

In Latin American cities, higher socioeconomic levels are usually associated with better living conditions which determine socio-spatial segregation. This research assessed territorial equity in the urban area of Medellín (Antioquia, Colombia), at the neighborhood scale using a spatial correlation index and univariate and bivariate dispersion plots. The variables were public space, natural hazards, and air quality; they were correlated to the prevailing socioeconomic level in the population. It was found that spatial distribution of the variables in the city of Medellín does not correlate to socioeconomic conditions, both in desirable variables such as public space which results from recent interventions aimed at balancing territorial distribution; and also in pollution and natural hazard variables that imply important management challenges throughout the city.

Key words: Socio-spatial segregation; Spatial autocorrelation; Value of urban housing; Moran index

Cita sugerida: Cárdenas, M.F.; Escobar, J.F. y Gutiérrez, K. (2020). Equidad territorial en Medellín: espacio público, amenazas naturales y calidad del aires. Estudios Socioterritoriales. Revista de Geografía, (27), 046. DOI: //https://doi.org/10.37.838/unicen/est.27-046 


\section{INTRODUCCIÓN}

La localización de la vivienda en el espacio urbano tiene influencia en las condiciones de vida de las personas, de acuerdo con las condiciones ambientales, la accesibilidad a infraestructura, equipamientos y al espacio público que posea; esto es, las posibilidades de acceso al consumo de bienes y servicios urbanos. Lo anterior hace parte de los factores que determinan la estratificación socioeconómica de las comunidades urbanas, y constituyen procesos que están configurados por la naturaleza intrínseca de la misma urbanidad (Soja, 2010). En contraposición a la resultante segregación residencial que ha sido especialmente marcada en las ciudades latinoamericanas, surge el concepto de equidad territorial, con la cual se designa una configuración espacial que aseguraría a todos las mismas condiciones de acceso a los servicios públicos, al empleo y a las diversas ventajas de la vida en sociedad (Bret, Gervais-Lambony y Hancock, 2016).

Los movimientos por la justicia ambiental se originaron en Estados Unidos en la década de 1960, resaltando el carácter socialmente desigual de las condiciones de acceso a la protección ambiental. En las últimas décadas este concepto ha emergido con notable impulso como principio necesario para valorar situaciones geográficas y para orientar la toma de decisiones territoriales (Moreno Jiménez, 2010). El concepto de justicia ambiental, estrechamente ligado al de justicia territorial, busca determinar si hay discriminación o segregación espacial de los grupos poblacionales, en este caso, con respecto a condiciones ambientales adecuadas o deseables. Ello puede traducirse en situaciones de equidad o inequidad territorial en cuanto al acceso a bienes y servicios o con relación a la calidad del medio que habitan ciertos grupos de personas.

En la actualidad existe consenso en torno a que las condiciones de inequidad son una amenaza para la sostenibilidad planetaria (Medellín, EDU y BID, 2014). En concordan- cia con esta problemática detectada en gran parte de las ciudades latinoamericanas, en la nueva agenda urbana se planteó como uno de los compromisos:
promover una ordenación territorial y urbana integrada, incluidas las ampliaciones urbanas planificadas sobre la base de los principios de equidad, el uso eficaz y sostenible de la tierra y los recursos naturales (...) y los múltiples usos del espacio, así como los usos sociales y económicos mixtos en las zonas construidas... (ONU, 2016, p. 17)

Este planteamiento implica una evaluación de las políticas de redistribución a través de la inversión de recursos en las ciudades, como elemento de justicia social, mediante actuaciones públicas que atenúen los contrastes de localización (Ojeda Casares, de Oliveira Neves, Schröder y Hurtado Rodríguez, 2008), en la búsqueda de una equidad territorial en las condiciones de la población que habita las ciudades.

El derecho a la ciudad se concreta, entre otras cosas, en el acceso a lo público, es decir, a las redes del espacio colectivo (educación, bienestar, salud, recreación, entre otras) y a las redes interdependientes de las centralidades metropolitanas. En otras palabras, los ciudadanos tienen el derecho a tener una estructura urbana equilibrada y solidaria (Talavera-Dávila, 2017). Lo anterior se formaliza a través de los espacios públicos que, como lugares de encuentro colectivo son la esencia de la ciudad, ya que las relaciones entre los habitantes y entre el poder y la ciudadanía se materializan, se expresan en la conformación de las calles, plazas, parques, lugares de encuentro ciudadano, en los monumentos (Borja y Muxí, 2000). En el espacio público los individuos adquieren y exhiben distintas formas de interacción social $y$, por tanto, se constituye en un medio para asegurar la equidad territorial en tanto que les permite a los ciudadanos realizarse social y ambientalmente, siempre y cuando este espacio público sea efectivo, es decir, que se pueda utilizar permanentemente, a cualquier hora del día y por diferentes motivos (Gehl, 2014).

La problemática ambiental es un reto con- 
temporáneo para las ciudades, especialmente en aquellas donde los procesos de urbanización y crecimiento poblacional ocurren aceleradamente (Delgado Campos y Rentería, 2012), y en muchos casos de manera no planificada, como es la situación de Medellín. Teniendo en cuenta lo anterior, se realizó un análisis de la distribución espacial, en la zona urbana de la ciudad, de tres variables que permiten la aproximación a una idea de la calidad ambiental de los barrios de Medellín, bajo el enfoque de la equidad territorial:

El espacio público hace parte de las condiciones medio ambientales deseables de una ciudad, ya que en este se incorporan gran parte de las áreas verdes y de las zonas arboladas dentro de la mancha urbana, es decir, los recursos naturales en las ciudades, que contienen los ecosistemas urbanos asociados a algunos servicios ambientales como la absorción de gases de efecto invernadero y de contaminantes del aire, reducción del efecto de islas de calor (ONU, 2016), entre otros. Adicionalmente, el espacio público es un espacio diferenciado que posibilita a los ciudadanos usarlo para compartir, socializar, convivir y construir comunidad (Berroeta, Carvalho y di Masso, 2016; Ochoa, 2011; Skewes Vodanovic, Trujillo Bilbao y Guerra Maldonado (2017), lo que incide positivamente en las condiciones y en la calidad de vida de la población (Borja y Muxí, 2000; Daza, 2008; Gómez, 2005; Páramo, 2010; Vergara et al., 2015).

Las amenazas naturales constituyen una situación no deseable en el territorio ya que imponen restricciones para el asentamiento poblacional o, en caso de no atenderse dicha limitación, como ocurre en muchos barrios de origen espontáneo en la ciudad, se convierten en un riesgo para la población y en un reto para la administración local que debe mitigar, reducir o gestionar ese riesgo. En el valle donde se asienta Medellín las amenazas naturales son variadas, algunas de ellas son de carácter no mitigable. Esto se debe a las características físicas del terreno y a la forma en que se dio el proceso de ocupación de sus laderas y cauces. Entre las amenazas estudiadas y evidenciadas en este territorio se cuentan los movimientos en masa, los sismos, las inundaciones y las avenidas torrenciales; las cuales tienen un alto potencial de daño por las condiciones de vulnerabilidad en que se asientan muchos de los pobladores (Caballero, 2011). Para resaltar la importancia de considerar estas situaciones en el territorio de Medellín, cabe anotar que en todas las temporadas de lluvias se presentan emergencias de este tipo, aunque pueden mencionarse algunos de los mayores eventos registrados en la ciudad, tales como una avenida torrencial de la quebrada La Iguaná el 23 de abril de 1880 que destruyó el poblado de Aná; el deslizamiento de Villatina ocurrido el 27 de septiembre de 1987, que ocasionó la muerte de unas 500 personas, destruyó unas 100 viviendas y dejó cerca de 1.700 damnificados; un flujo de tierra en el barrio El Socorro el 13 de mayo de 2008 que destruyó 20 viviendas y dejó 27 personas faIlecidas; y en el barrio El Poblado, urbanización Altoverde, el 16 de noviembre de 2008 cuando colapsaron seis viviendas y fallecieron 12 personas por un movimiento en masa (Hermelin, Echeverri, y Giraldo, 2010).

La polución del aire se ha reconocido en los últimos años como una de las problemáticas urbanas que más afectan la salud de la población, por lo que las naciones se han comprometido a mantener la calidad del aire dentro de los estándares indicados por la OMS (ONU, 2016); lo cual también va de la mano con los espacios verdes públicos de buena calidad. En Medellín, debido a la concentración de fuentes fijas y móviles de emisión de gases y material particulado, sumado a la geomorfología del valle en el cual se localiza la ciudad, en los últimos años se han venido presentando crecientes 
problemas relacionados con la calidad del aire (AMVA, 2016).

Con el ánimo de evaluar la equidad territorial en la ciudad de Medellín relacionando las variables seleccionadas con el valor de venta de los inmuebles para vivienda; se recurrió a los análisis de autocorrelación y de dependencia espacial; metodología desarrollada en el marco de la geografía, al estudiar la relación del hombre con el medio (Buzai, 2016), que permite estimar estadísticamente los niveles y la dirección de las relaciones entre variables espaciales.

La autocorrelación espacial aparece como consecuencia de la existencia de una relación funcional entre lo que ocurre en un punto determinado del espacio y lo que ocurre en otro lugar. Esta puede ser positiva o negativa, según el sentido de las relaciones encontradas, siendo positiva para relaciones directamente proporcionales, o negativa para comportamientos inversos, es decir, cuando unidades geográficas cercanas sean más disímiles entre ellas que entre regiones alejadas en el espacio. Asimismo, la autocorrelación espacial será nula cuando la variable analizada se distribuya de forma aleatoria (Moreno y Vayá, 2002).

\section{Metodolocía}

\section{SITIO DE ESTUDIO}

Este trabajo se realizó en la zona urbana de la ciudad de Medellín, tomando como unidad mínima de análisis el barrio. Medellín es la capital del departamento de Antioquia. Se asienta en la parte más ancha de la región natural conocida como Valle de Aburrá, en la Cordillera Central de los Andes a $1.475 \mathrm{~m}$ s.n.m., con una temperatura promedio de $24^{\circ} \mathrm{C}$ (Figura 1).

La ciudad tiene una extensión total de 380,2 $\mathrm{km} 2$, de los cuales $105 \mathrm{~km} 2$ corresponden a suelo urbano, $5,2 \mathrm{~km} 2$ son de suelo para expansión urbana y los $270 \mathrm{~km} 2$ restantes son de suelo rural (Alcaldía de Medellín, 2017). Según proyecciones del Departamento Administrativo Nacional de Estadística -DANE-, la población de Medellín a 2015 era de 2.464.322 habitantes, lo que la hace la segunda ciudad más poblada de Colombia. La ciudad se distribuye político-administrativamente en dieciseis comunas y cinco corregimientos. A su vez, las comunas se subdividen en barrios que suman 249 (Alcaldía de Medellín, 2017).

De acuerdo con el informe de calidad de vida de la ciudad, en 2017 la desigualdad por ingresos fue muy alta, con un valor de 0,52 en el índice de Gini (Medellín Cómo Vamos, 2018). Para el año 2017 se reporta un 14,2\% de la población en situación de pobreza, y marcadas diferencias en el territorio en cuanto a las condiciones de vida: mientras que la comuna $14 \mathrm{El}$ Poblado presenta las mejores con un indicador de 75,3/100; la comuna 1 Popular fue la de menores condiciones de vida con 34,8/100 (Medellín Cómo Vamos, 2018). No obstante, las diferencias en el territorio no solamente se manifiestan en las condiciones de vida y en los costos de acceso a la vivienda, sino también en las formas constructivas y en las densidades poblacionales. Para verificar lo antes dicho, se relacionó el valor medio de la vivienda por comuna, con las densidades poblacionales (habitantes $/ \mathrm{km} 2$ ) calculadas a partir de la información poblacional del DANE y el área total de cada comuna. 
Figura 1. Esquema de localización de a) Colombia, donde el color rojo señala el departamento de Antioquia; b) el Departamento de Antioquia, donde el color rojo señala el municipio de Medellín; y c) el municipio de Medellín, donde el color rojo señala el área urbana

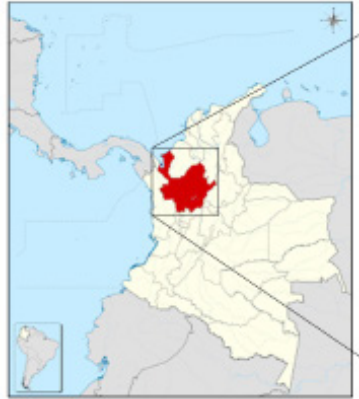

a) Colombia

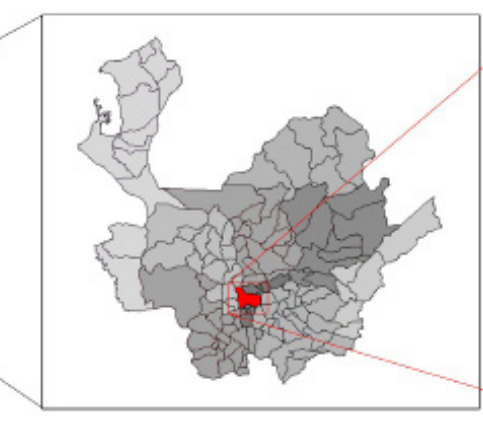

b) Departamento de Antioquia

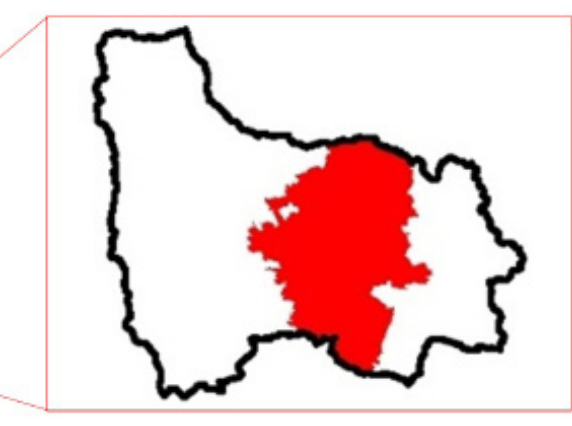

c) Municipio de Medellin

Fuente: elaboración personal

\section{VARIABLES EVALUADAS}

Para realizar el ejercicio se emplearon tres variables que pueden señalar algunas de las condiciones biofísicas y ambientales de los barrios: las áreas de espacio público, las áreas expuestas a algún tipo de amenaza natural y la calidad del aire expresada en la concentración promedio (en $\mu \mathrm{g} / \mathrm{m} 3$ ) de material particulado suspendido con diámetro inferior a 2,5 micras o PM2.5. Para contrastar las variables anteriores con una variable que de cuenta del nivel socioeconómico de la población en cada sector, se utilizó el valor promedio del metro cuadrado comercial de los inmuebles para uso residencial. Todo el procesamiento cartográfico se realizó por medio del software de procesamiento de información geográfica ArcGIS 10.4 de ESRI.

\section{ESPACIO PÚBLICO}

El municipio de Medellín en su Plan de Ordenamiento Territorial -POT- vigente ((Alcaldía de Medellín, 2014), estableció que el espacio público efectivo es el espacio público de carácter permanente conformado por los parques, plazas, plazoletas y zonas verdes. Para deter- minar la distribución espacial del espacio público urbano de Medellín, se tomó como referencia la cartografía del subsistema de espacio público de encuentro y esparcimiento generada y puesta a disposición por la Alcaldía de Medellín a través del geoportal web de Open Data $^{1}$, -en adelante, geoportal- que reporta 790,7 ha de espacio público urbano (Figura 2). Adicionalmente, se incluyeron las intervenciones recientes en el espacio público que aún no estaban incorporadas en la cartografía oficial de Medellín, tales como las denominadas unidades de vida articulada -UVA- y la etapa inicial de Parques del Río, con lo cual se sumaron otras 46,5 ha, para un total de 837,2 ha de espacio público, lo que resulta en un indicador de 3,4 m2/habitante. La distribución espacial se evaluó mediante el indicador de área de espacio público para cada barrio (en m2) / área del barrio (en km2). También se calculó el indicador más utilizado para esta variable que es área de espacio público (en m2) / habitante, para la escala barrial.

1 Se trata de un portal oficial de la Alcaldía de Medellín que pone a disposición del público en general información producida por las entidades gubernamentales: https://geomedellin-m-medellin.opendata.arcgis.com/ 
Figura 2. Espacio público en Medellín

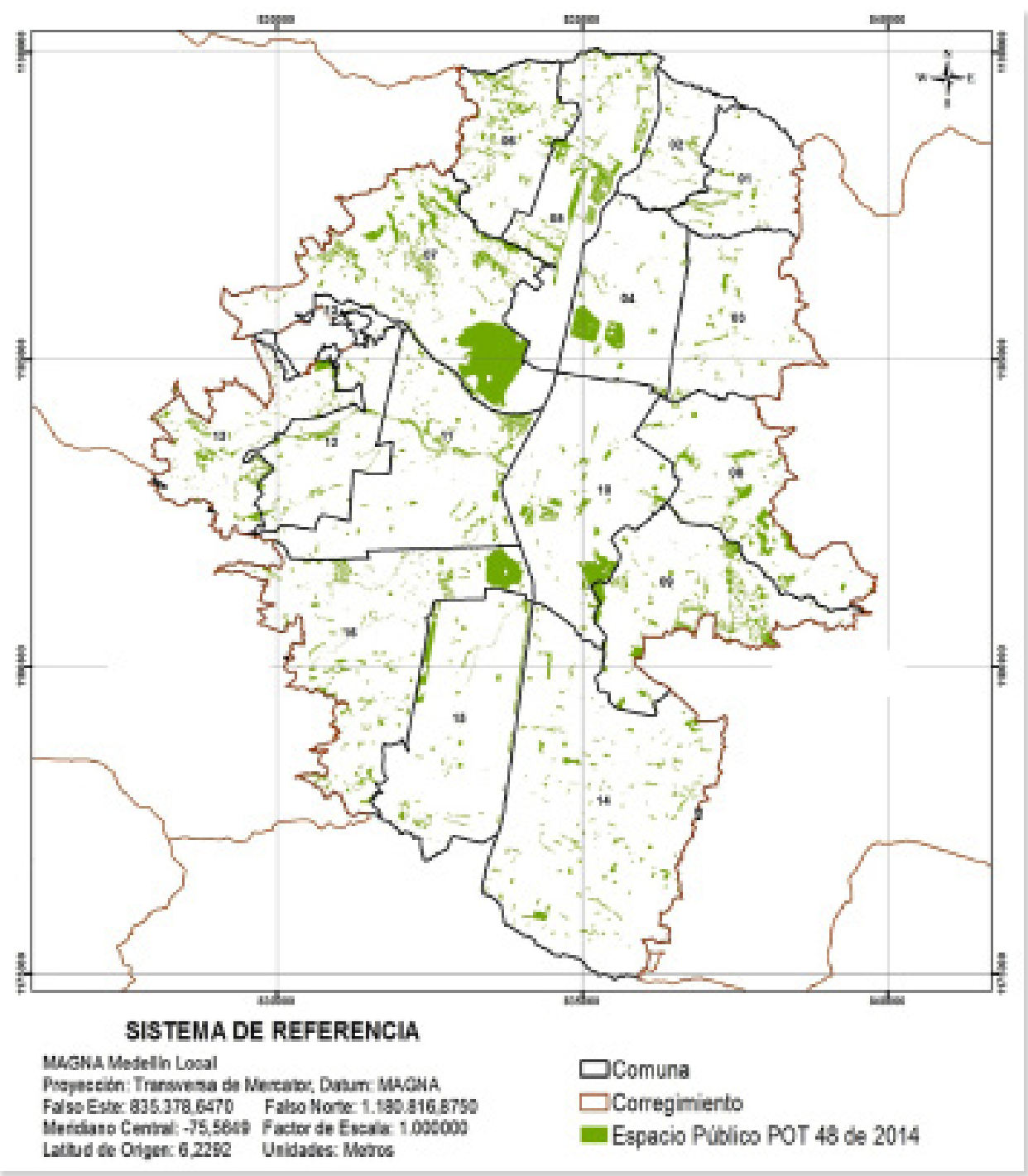

Fuente: elaboración personal con base en datos del POT, a partir de Geoportal y Medellín (2014)

\section{AMENAZAS NATURALES}

Para esta variable se tomó la información del geoportal, ya que, como parte de la revisión del POT de Medellín (2014), se evaluaron las amenazas naturales en el territorio y el riesgo que estas representan. Según la cartografía del POT de Medellín, el área urbana del municipio tiene 26,84 ha en algún tipo de amena- za, dentro de los cuales predomina el de movimientos en masa (16,7 ha), seguido por las avenidas torrenciales que amenazan 6,42 ha $y$ las inundaciones amenazan 3,72 ha del territorio (Figura 3). A partir de esta información, se determinaron las áreas con algún tipo de amenaza en cada barrio, y se calculó el indicador de área bajo amenaza en el barrio (en m2) / área del barrio (en km2). 
Figura 3. Áreas con condiciones de amenaza y riesgo en la ciudad de Medellín

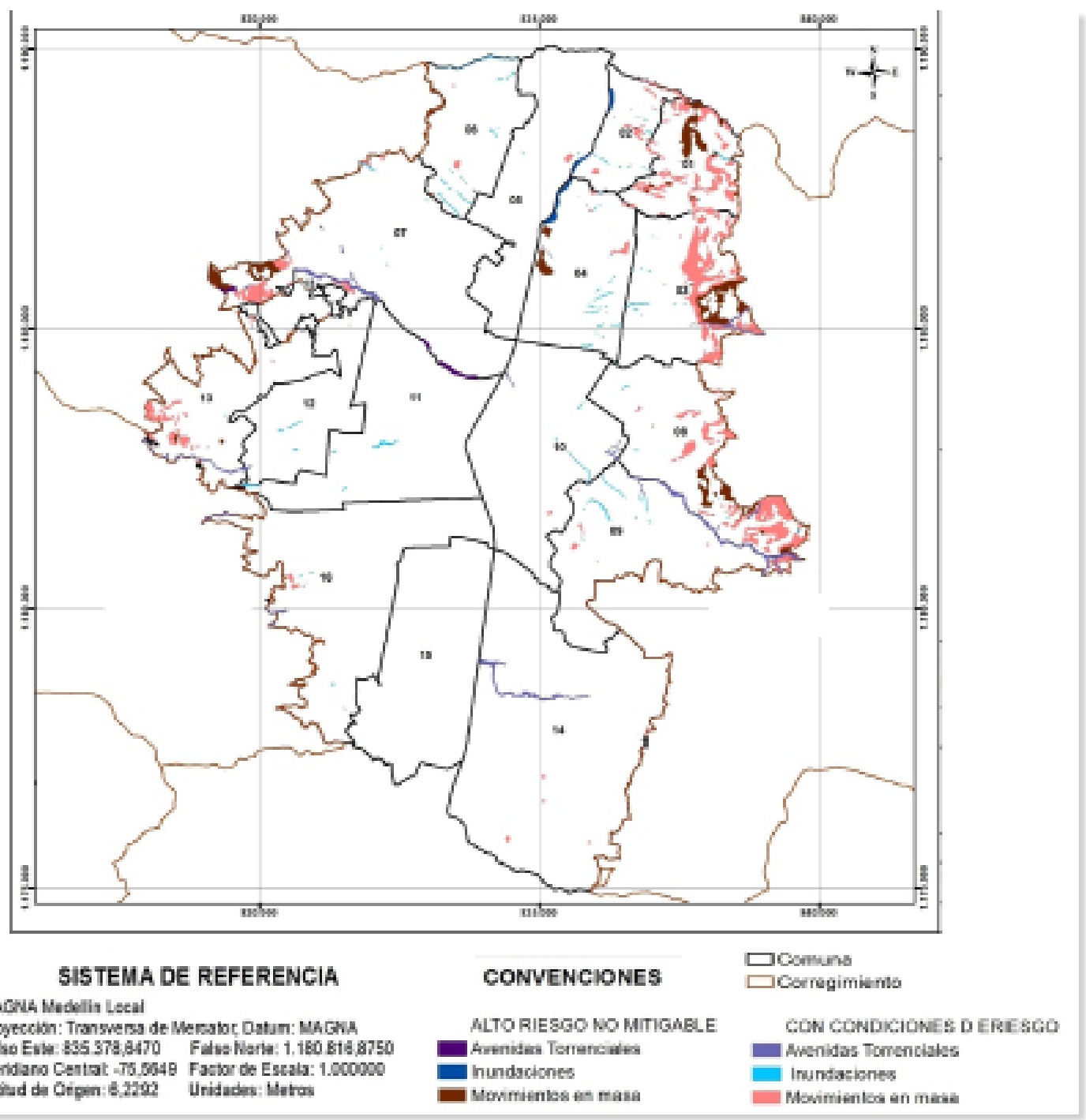

Fuente: elaboración personal con base en Medellín (2014)

\section{CALIDAD DEL AIRE}

La ciudad de Medellín y su área metropolitana monitorea las condiciones del aire a través de un conjunto de estaciones permanentes y otras móviles, que registran la información de distintas variables a escala horaria. Estos datos se encuentran disponibles en la página del SIATA². Aunque Medellín solo tiene nueve estaciones permanen-

2 Sistema de Alerta Temprana del Área Metropolitana del Valle de Aburrá, encargado de monitorear y gestionar la información meteorológica e hidrológica del valle. La información está disponible en www.siata.gov.co tes en su jurisdicción, para hacer la interpolación se consideraron 12 estaciones en total, incluyendo dos más localizadas al sur y una en el norte (Figura 4). La información empleada para este ejercicio fue el PM2.5 ya que se considera el material más dañino para la salud humana (OMS, 2016). Se descargaron todos los datos medidos durante el año 2018. La interpolación se realizó con el promedio de los datos en $\mu \mathrm{g} / \mathrm{m} 3$, empleando el método de kriging en el software ArcGis, a partir de lo cual se obtuvo el valor promedio de PM2.5 para cada barrio, para el año 2018. 
Figura 4. Estaciones de calidad del aire e interpolación de mediciones promedio de concentración de PM2.5 en $\mu \mathrm{g} / \mathrm{m} 3$ para el área urbana de Medellín

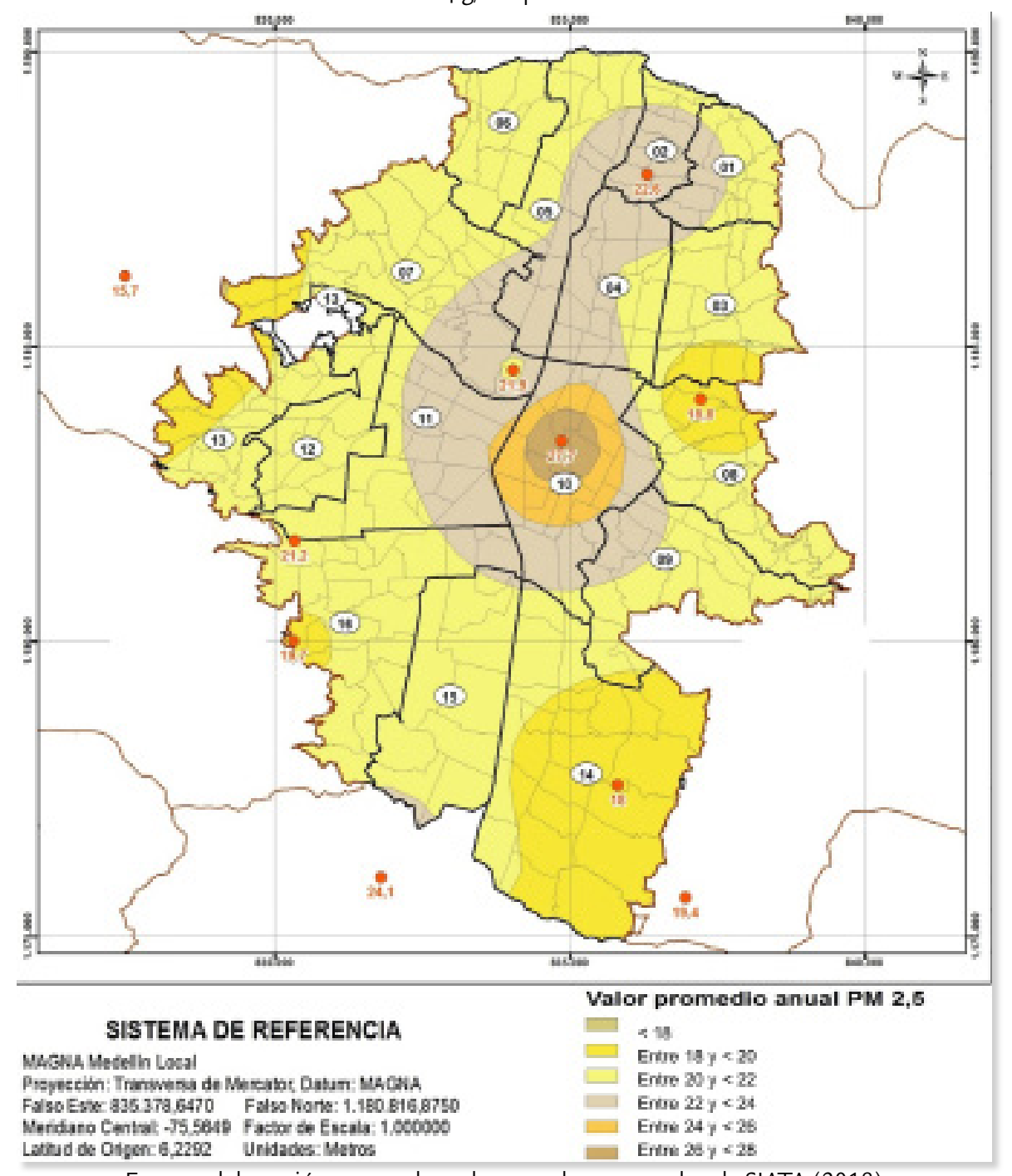

Fuente: elaboración personal con base en datos tomados de SIATA (2018)

\section{DISTRIBUCIÓN ESPACIAL DEL VALOR DEL SUELO}

Como una aproximación a la renta diferencial en los espacios de la ciudad, se consideró el valor de venta de las viviendas nuevas. Para ello se usó la información del Observatorio Inmobiliario de Medellín OIME, actualizada a febrero de 2018, de la cual se obtuvieron 2.115 registros de inmuebles en venta con su valor por metro cuadrado $(\$ / \mathrm{m} 2)$ y su ubicación geográfi$\mathrm{ca}^{3}$. Con la información obtenida se realizó una interpolación espacial con el método de kriging por distancia, usando el software ArcGis, a fin de generar un mapa con valores continuos para el

3 Tomado de http://catastrooime.blogspot.com/ área urbana de Medellín (Figura 5). Con los valores resultantes de este mapa, se sacó el valor promedio para cada barrio, ponderando por el área que ocupaba cada rango de valor del suelo, y tomando para el cálculo, el valor medio de cada rango, en pesos ${ }^{4}$ por metro cuadrado.

Asimismo, se hizo un análisis para determinar si hay una relación entre el valor medio de la vivienda y la densidad poblacional, calculada con base en la población proyectada por el DANE para 2018. Dado el gran número de barrios de la ciudad, este se hizo a escala de comuna.

4 El peso colombiano tiene una tasa de cambio a dólares de 1 US\$ = col \$3.150. Valor medio a inicios del año 2019. 
Figura 5. Distribución espacial de la renta media en la ciudad, expresada como valor promedio de los inmuebles para vivienda en $\$ / \mathrm{m} 2$

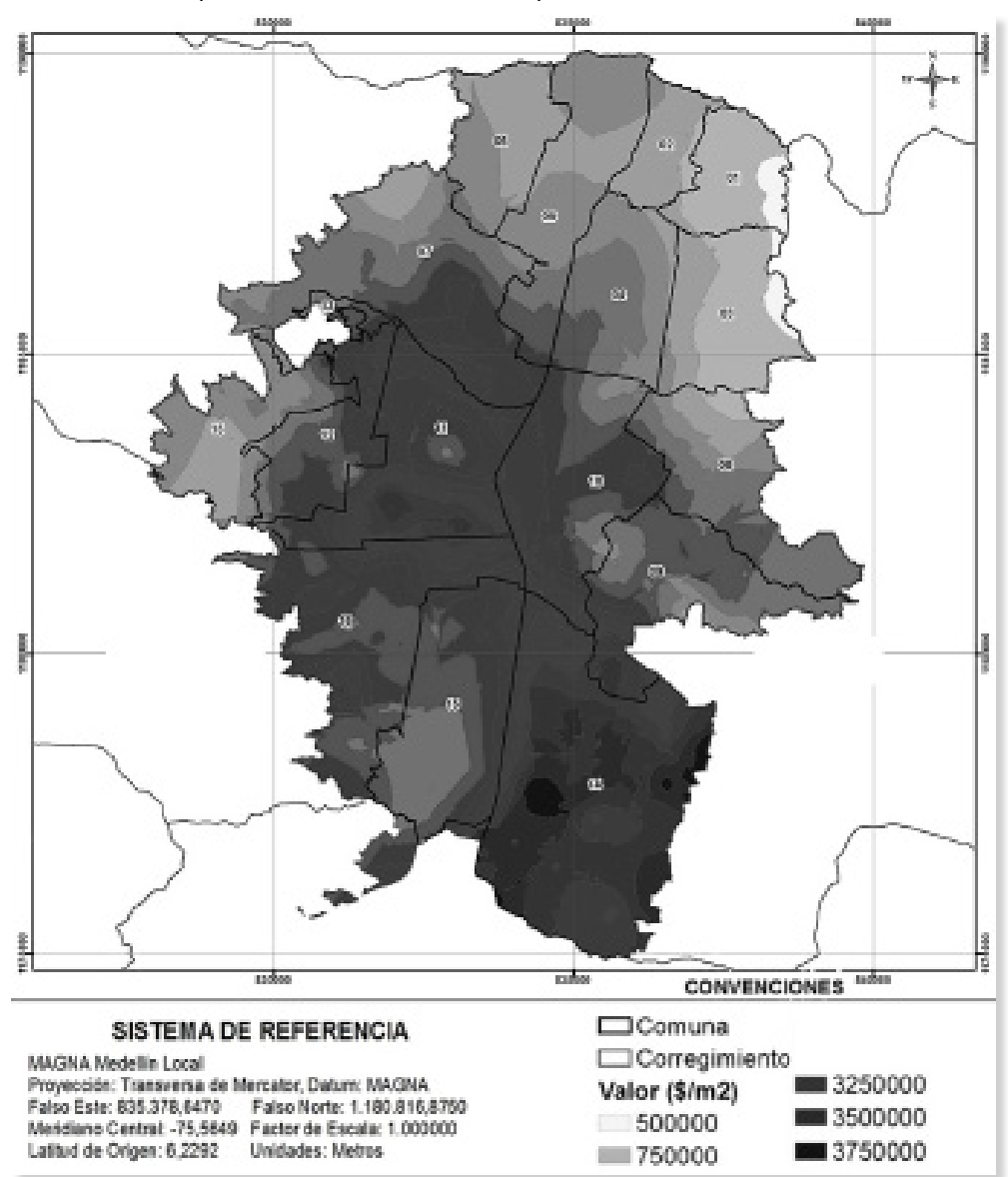

Fuente: elaboración personal con base en datos de Subsecretaría de Catastro (2018)

\section{AUTOCORRELACIÓN ESPACIAL DE LAS VARIABLES}

Una forma de comparar visualmente las distribuciones espaciales de las variables es por medio de superposición cartográfica en SIG. También hay herramientas estadísticas simples como el diagrama de dispersión; o estadísticos que fueron diseñados para medir la autocorrelación espacial.

Una de las herramientas más utilizadas con este fin es el índice I de Moran, el cual es una adaptación de una medida de correlación no-espacial a un contexto espacial y se aplica normalmente a unidades espaciales donde hay disponibilidad de información en forma de razones o intervalos (Acevedo y Velásquez, 2008). Para este ejercicio se utilizó el índice I de Moran global, cuya expresión numérica se calcula según la siguiente ecuación (Moreno y Vayá, 2002).

$$
I g=\frac{N}{S o} \cdot \frac{\sum_{i j}^{N} w i j(x i-X)(x j-X)}{\sum_{i=1}^{n}(x i-X)^{2}}
$$

Donde,

xi: valor de la variable $\mathrm{x}$ en la región $\mathrm{i}$

$x j$ : valor de la variable $x$ en las regiones $j$, vecinos a la región $i$

$X$ : media muestral de la variable $x$

wij: pesos de la matriz $\mathrm{W}$

$\mathrm{N}$ : tamaño muestral

S0: Sumatoria de los pesos $\mathrm{w}$

La matriz $W$ es una matriz cuadrada con ceros en su diagonal, que refleja las relaciones de vecindad entre las unidades de análisis, en este caso, los barrios de Medellín. La matriz se llenó con uno cuando se trata de unidades vecinas y con cero cuando no lo son; por lo cual esta matriz ilustra la relación que tiene cada uno de los barrios de la ciudad con los demás. 
Asimismo, se analizaron los datos con los gráficos de dispersión. En este tipo de gráficos, en el eje de las abscisas aparecen los valores estandarizados de una variable para cada unidad espacial y en el eje de las ordenadas se encuentran los valores estandarizados del promedio de los valores de las unidades espaciales vecinas para la misma variable (univariada) o de una segunda variable (bivariada). En ambos casos, la recta de regresión lineal muestra el grado de asociación entre la variable y los valores contiguos de la misma u otra variable considerada (Buzai et al., 2015). El valor de la pendiente, que puede interpretarse como el coeficiente de correlación de Pearson, corresponde en este análisis al índice I de Moran. El diagrama divide el espacio en cuatro tipos de relación espacial, que pueden analizarse a partir de la media de cada variable, y si los valores están por encima o por debajo de esta media en una o en las dos variables estudiadas, para el caso del análisis bivariado.

Aunque en estudios similares utilizan cálculos de índices agregados, por ejemplo de calidad de vida (Lucero y Celemín, 2008) o de lo que denominan los autores determinantes sociales de la salud (Buzai y Villerías Alarcón, 2018) para luego evaluar su correlación espacial, en este ejercicio se optó por analizar las autocorrelaciones de las variables y las relaciones entre las variables físicas del territorio con el valor del suelo para viviendas, a fin de evaluar el comportamiento de cada una de ellas por separado.

\section{Resultados}

RELACIÓN ENTRE VALOR DE LA VIVIENDA Y DENSIDAD POBLACIONAL POR COMUNA

A partir de la distribución espacial del valor medio de la vivienda en cada comuna, se hizo un análisis preliminar con respecto a la densidad poblacional estimada para cada una. Para representar esta relación gráficamente, se organizaron las comunas de menor a mayor valor medio de las viviendas (Figura 6). Aunque la relación no es lineal, sí se nota que en aquellas porciones del territorio donde los costos son menores, las densidades poblacionales tienden a ser más altas. También vale resaltar que la comuna con menor densidad poblacional es la 10, ubicada en el centro tradicional de la ciudad y donde se concentran gran parte de las actividades comerciales y de servicios de escala municipal e incluso regional; lo que explica que sea baja la población asentada allí. Algo similar ocurre con la comuna 15 , que concentra espacios lúdicos, recreativos y de servicios, algunos de grandes extensiones.

Figura 6. Relación entre el valor medio de la vivienda y la densidad poblacional en las comunas de Medellín

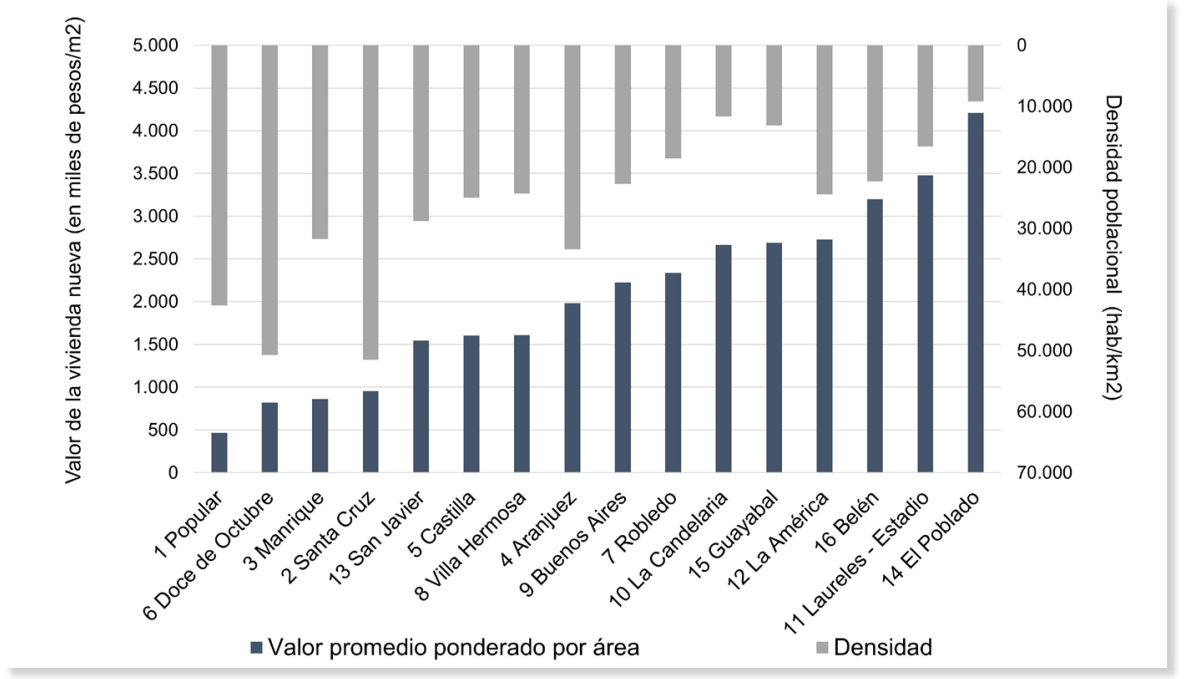

Fuente: elaboración personal 


\section{AUTOCORRELACIÓN ESPACIAL UNIVARIADA}

El análisis de autocorrelación espacial y el cálculo del índice I de Moran se realizaron para los 249 barrios y 13 zonas intersticiales ${ }^{5}$ que conforman el área urbana de Medellín. Estos se encuentran dentro de alguna de las 16 comunas (numeradas de 1 a 16) y su codificación inicia por el número de la comuna a la que pertenecen. Así, en los resultados se presentan los códigos de cada barrio.

De las cuatro variables analizadas, solamente dos presentaron autocorrelación espacial significativa, lo que indica que los valores obtenidos

5 Hace referencia a polígonos institucionales o áreas privadas de una extensión tal que en mapa aparecen separadas del barrio, tales como algunas universidades, un club privado, el centro administrativo entre otros. para un barrio están correlacionados con los valores de los barrios vecinos (Figura 7). De las variables ambientales, fue el PM2.5 la única significativa (Figura 7a), lo cual tiene sentido ya que las fuentes de polución están concentradas en ciertas áreas de la ciudad y se dispersan en el espacio según la dirección de las corrientes de aire. La otra variable que tiene autocorrelación significativa es el valor medio de la vivienda. Es claro que la cercanía a ciertos sectores o a determinadas infraestructuras de escala municipal tiene efectos positivos o negativos sobre los valores de los inmuebles, los cuales se diluyen al incrementar la distancia. En este caso la autocorrelación no solo es significativa, sino que es muy fuerte, como lo indica el coeficiente de determinación y como puede verse en la Figura 7b y en la Tabla 1.

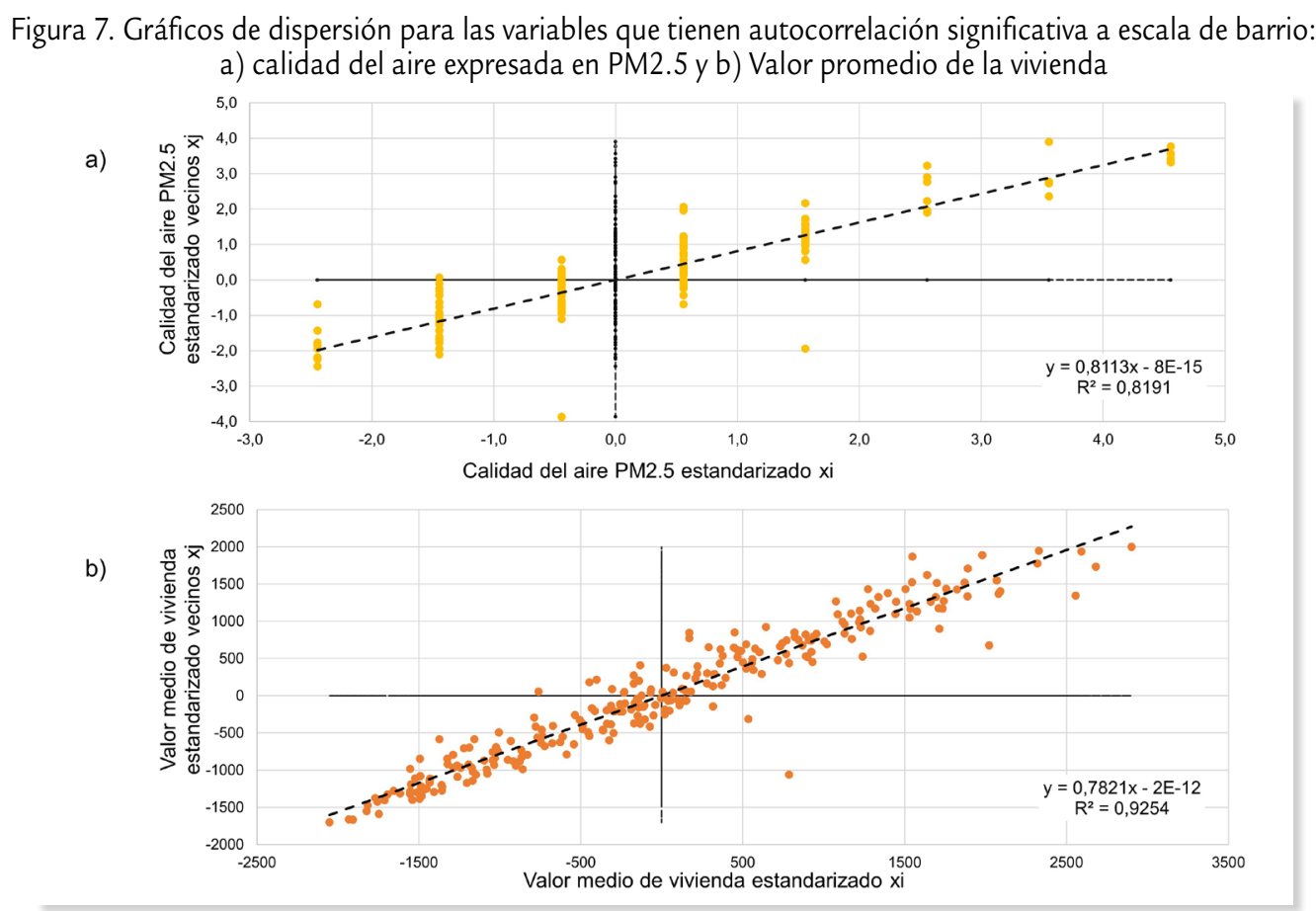

Fuente: elaboración personal

\section{AUTOCORRELACIÓN ESPACIAL BIVARIADA}

La Tabla 1 presenta los resultados obtenidos al relacionar el valor de cada una de las variables a escala de barrio, con respecto a los valores promedios de los barrios vecinos para esa misma variable -autocorrelación-. De igual forma, presenta en la última columna los re- sultados del índice I de Moran resultantes al correlacionar cada una de las variables ambientales con respecto a la variable socioeconómica, representada por el valor medio por metro cuadrado de la vivienda en cada barrio. En este último caso, es posible notar que los valores son cercanos a cero, lo cual indica que 
el par de variables analizadas tienen una distribución completamente aleatoria. En otras palabras, las áreas de espacio público, las zonas con amenazas naturales y la calidad del aire según el PM2.5, se distribuyen en el espacio urbano de Medellín de manera totalmente independiente del valor de renta o el valor del suelo, expresado como el valor de venta de inmuebles para vivienda.

A pesar de lo anterior, los gráficos de dispersión bivariados permiten identificar cuáles son los barrios que presentan los valores más alejados de las medias para cada una de las variables analizadas (Figura 8).

Tabla 1. Resultados del índice I de Moran univariado (autocorrelación) para las cuatro variables evaluadas, y de las tres variables ambientales con respecto al nivel socioeconómico promedio a escala de barrio (bivariado)

\begin{tabular}{lcr}
\hline \multirow{2}{*}{ Variable } & \multicolumn{2}{c}{ Índice I de Moran } \\
\cline { 2 - 3 } & Univariado & $\begin{array}{c}\text { Bivariado con respecto } \\
\text { al valor de la vivienda }\end{array}$ \\
\hline Espacio público & 0,0301 & $-0,0617$ \\
\hline Amenazas naturales & 0,6205 & 0,0285 \\
\hline Calidad del aire & 0,8113 & 0,0372 \\
\hline Valor de vivienda & 0,7408 & \\
\hline
\end{tabular}

Fuente: elaboración personal

Figura 8. Gráficos de dispersión bivariados, de las variables ambientales con respecto al valor medio de la vivienda:

a) Espacio público; b) áreas con amenazas naturales; y c) calidad del aire según PM2.5.

El número que acompaña cada punto corresponde al código del barrio que representa

a)

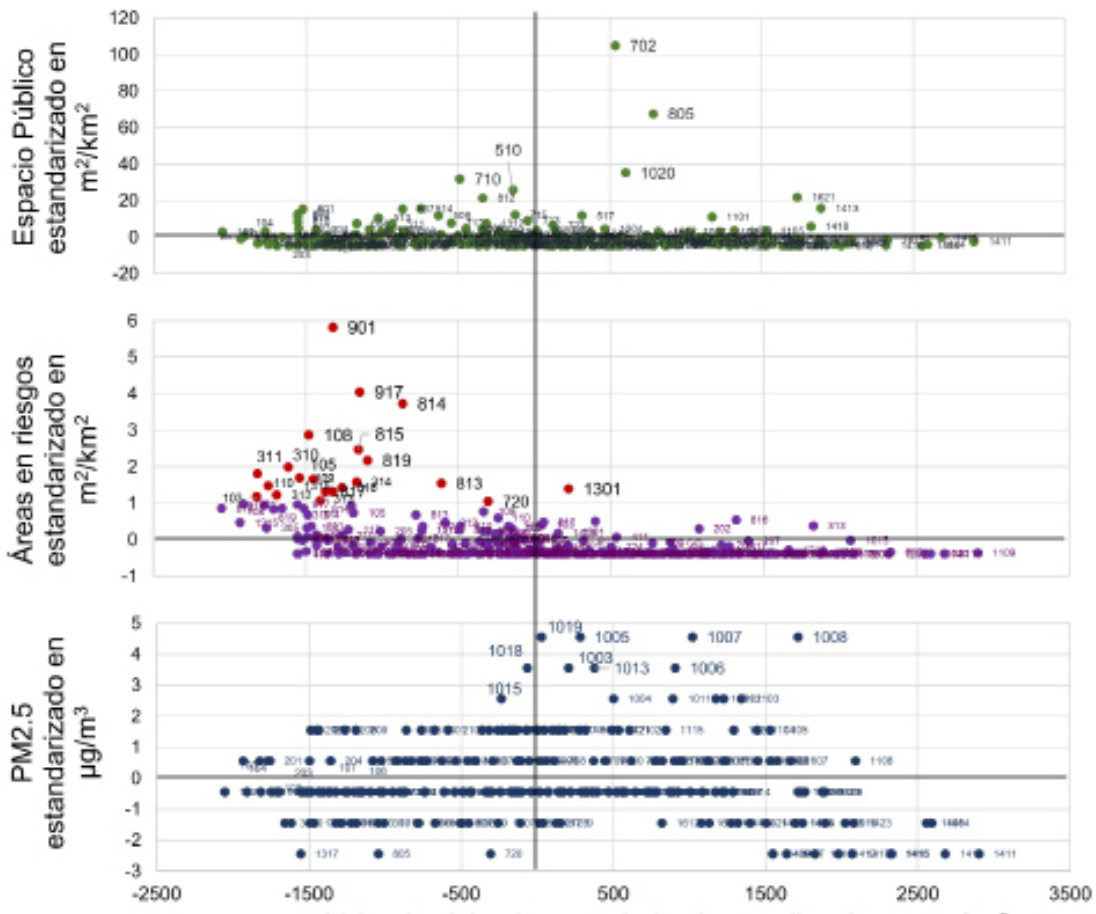

Valor de vivienda estandarizado en miles de pesos $/ \mathrm{m}^{2}$

Fuente: elaboración personal 
Del diagrama de dispersión de espacio público (Figura 8a) se destacan cinco barrios que tienen áreas con esta destinación muy por encima del promedio, las cuales corresponden, en parte, a aquellos que contienen los cerros tutelares de la ciudad y que constituyen los parches de espacio público más grandes, que se localizan en las comunas 7, 10 y 16; así como zonas verdes en la comuna 8 y un conjunto de parques de la comuna 5, como puede observarse en la Figura 2.

Del análisis de amenazas, es claro que estos tienden a concentrarse en unas áreas específicas que están asociadas a fuertes pendientes y a corrientes de agua con condiciones de torrencialidad. En este caso, a pesar de que los resultados de correlación espacial indican que no hay una dependencia espacial entre la amenaza natural y el valor de las viviendas, se nota en la Figura $8 \mathrm{~b}$ que la mayor parte de los barrios que tienen una exposición a amenazas, se concentran en el cuadrante IV que indica que su renta o el valor de las viviendas están por debajo del promedio.

La calidad del aire, como se nota en la Figura 4 y se reafirma en la Figura $8 c$, presenta sus condiciones más críticas de PM2.5 en la zona centro de la ciudad, que corresponde a la comuna 10. Aunque esta es la comuna con menor densidad de habitantes por unidad de área (Figura 6), diariamente transitan por allí miles de personas que están sometidas a condiciones ambientales deterioradas; aunque es justamente el alto flujo vehicular una de las razones de la concentración de la polución en esta zona.

\section{DisCUSIÓN}

Medellín a través de su historia ha tenido procesos de concentración, traslado en bloque de las familias con mejores recursos hacia ciertos sectores que no necesariamente ofrecen las mejores condiciones de conexión o de acceso a infraestructuras, pero que sí gozan de un alto valor simbólico o de prestigio (Cárdenas y Agudelo, 2013). También ha presentado procesos de gentrificación y otros que, en todo caso, han resultado en la expulsión de la población con menores recursos hacia las periferias, como bien lo explican Sabatini, Cáceres y Cerda (2001) para América Latina, y como se aprecia en el mapa de la Figura 5. En muchos casos, familias con escasos recursos deben conseguir donde habitar, lo que las lleva a localizarse de manera informal en áreas expuestas a amenazas naturales, lo que explica el crecimiento urbano sobre laderas inestables, que luego se consolidan y se formalizan.

La distribución desigual de la población en el territorio se explica, en parte, por el valor del suelo o la posibilidad de acceder a inmuebles en los distintos barrios. En Medellín, las personas que habitan zonas de nivel socioeconómico medio-bajo y bajo (estratos 1,2 y 3$)^{6}$ representan el $80 \%$ de la población, mientras que los sectores con niveles medio y alto suman $20 \%$. Las tipologías constructivas en los barrios con menor valor del suelo muestran predominio de casas de dos o tres pisos, con pocos espacios destinados a parqueaderos, antejardines o patios, ya que se trata de aprovechar al máximo el territorio. En esta misma lógica, los barrios espontáneos o autoconstruidos rara vez destinan espacios para calles, equipamientos y espacios públicos; por lo que las intervenciones de la administración municipal en sus programas de mejoramiento integral de barrios buscan generar este tipo de obras, de manera que se equilibren un poco la oferta con la demanda de servicios (Alcaldía de Medellín y BID, 2011). Esta manera de construir ciudad explica también las densidades poblacionales más altas que se encuentran en barrios con viviendas más baratas, que tienen una mayor ocupación del suelo; lo cual no necesariamente señala condiciones de hacinamiento o condiciones de vida precarias en sus habitantes. En contraste, sectores como la comuna 14 de Medellín, en donde predominan construcciones en altura para vivienda, al igual que para usos institucionales, comerciales y empresariales, presenta una de las densidades

6 En Colombia las viviendas se clasifican en estratos numerados de 1 a 6 , que indican el nivel socioeconómico predominante donde 1 equivale a bajo-bajo, 4 señala un nivel medio y 6 se refiere a nivel alto, en función de características de los inmuebles como extensión, acabados, materiales, accesibilidad y cercanía a infraestructuras y equipamientos, entre otros. 
más bajas de la ciudad, ya que entre edificios hay espacios libres que corresponden a zonas verdes privadas (situadas dentro de las unidades residenciales y con acceso restringido), zonas de retiro a las corrientes de agua, vías, senderos, equipamientos y complementos. La condición descrita en la comuna 14 El Poblado permite también aclarar que, aunque tenga más zonas verdes, bastante arbolado y áreas abiertas, en el cálculo del índice de espacio público no se destaca sobre los demás sectores de la ciudad porque dichas áreas no están disponibles para el uso y aprovechamiento de la ciudadanía, sino exclusivamente para los habitantes de los edificios a los que pertenecen.

Con respecto a las amenazas naturales, la situación de Medellín coincide con lo reportado por Vásquez y Salgado (2009) para dos comunas en Chile, según lo cual hay una mayor exposición a las amenazas naturales en los sectores de bajos ingresos, aunque en las áreas con mayores niveles socioeconómicos de Medellín también aplica su hallazgo de que en las zonas con mayores ingresos puede darse una disminución de la vulnerabilidad física, mediante acciones de mitigación, intervenciones con infraestructura o dejando dichas áreas libres de construcciones permanentes, con lo cual se reduce el riesgo al que se expone la población.

Aunque lo ideal sería tener cero áreas urbanas expuestas a amenazas, dadas las condiciones de relieve, los factores climáticos, y la expansión urbana que ha ascendido por las laderas del valle y se ha consolidado, es una condición inevitable en Medellín. En consecuencia, además de trabajar sobre medidas de mitigación, en donde ello es posible, la ciudad ha venido fortaleciendo el Sistema de Alerta Temprana que ayuda a determinar los sitios donde pueden ocurrir emergencias a fin de alertar y evacuar a la comunidad del área afectada, con lo cual se ha logrado reducir la cifra de pérdidas humanas en los eventos naturales que ocurren.

Con todo, la historia de Medellín en las últimas décadas ha estado caracterizada por la utilización de la renovación urbana con miras a superar la desigualdad social a través de intervenciones físicas en el territorio, entre otras cosas, con énfasis en crear espacios públicos incluyentes y de calidad (Alcaldía de Medellín y BID, 2011). Entre este tipo de proyectos, se pueden resaltar los llamados Proyectos Urbanos Integrales -PUI-, diseñados como un instrumento de planeación e intervención física en zonas caracterizadas por altos índices de marginalidad, segregación, pobreza y violencia (Hermelin et al., 2010), por medio de los cuales se ha logrado la generación y el mejoramiento del espacio público en las comunas de estratos socioeconómicos más bajos, propiciando una consolidación urbanística en algunos sectores de la ciudad. Recientemente, Empresas Públicas de Medellín -EPM-, decidió aprovechar los espacios de almacenamiento de agua para ceder espacios públicos a los habitantes de Medellín, a partir de lo que surgieron las UVA (Valencia, 2016). La construcción del megaproyecto Parques del Río pretende cambiar la concepción del espacio público en la ciudad, potenciando el río MedeIlín como el eje natural de esta área metropolitana(Sáenz, 2016). Lo anterior evidencia que Medellín ha apostado por transformarse y superar la desigualdad social a través del espacio público, entre otros aspectos.

Al contrastar los resultados obtenidos en este estudio con otros trabajos similares realizados para distintas ciudades, resalta esta como la única ciudad en la que, hasta ahora, los análisis revelan una distribución espacial equitativa de los atributos evaluados. Por ejemplo, con respecto al acceso a parques en Baltimore (Maryland, EE.UU), Boone, Buckley, Grove, Morgan y Sister (2009) encontraron que las personas blancas tenían acceso a mayores áreas de parques que los negros por lo que concluyen que las concepciones de la justicia deben ir más allá de la justicia distributiva ya que, en este caso, hay una discriminación desde el diseño de parques para blancos y para negros. En este mismo sentido, Landry y Chakraborty (2009) encontraron que la distribución de árboles en las calles de Tampa (Florida, EE.UU.) es inequitativa ya 
que es menor en los vecindarios que contienen mayor proporción de afroamericanos, residentes de bajos ingresos e inquilinos. De igual manera, en Mar del Plata (Buenos Aires, Argentina) Celemín (2012) encontró que las dimensiones ambiental y socioeconómica de la ciudad están interrelacionadas, ya que las problemáticas ambientales están concentradas en los sectores periféricos de la ciudad donde residen las personas de menores recursos. Con respecto a esta misma ciudad, pero producto de otro análisis, Lucero y Celemín (2008) afirman que los resultados encontrados "dejan ver la intensidad de la separación y ruptura en la construcción de la ciudad, ante la persistencia y consolidación de una estructura urbana polarizada socialmente y segregada espacialmente" (p. 10).

Con respecto a la calidad del aire, Moreno Jiménez (2007; 2013) analizó la distribución espacial del ruido y de la contaminación del aire con dióxido de nitrógeno en Madrid (España), de lo cual concluyó que no hay evidencia de un perjuicio para las áreas de menor renta, en lo concerniente a ruido nocturno inadecuado, lo cual coincide con los resultados obtenidos en el presente estudio. Sin embargo, con relación a la polución del aire, algunos de los grupos sociodemográficos vulnerables muestran una cierta propensión a la segregación espacial, como es el caso de los asiáticos (chinos), algunos africanos (marroquíes) y europeos (rumanos), aunque también encontró tendencias diversas en cuanto a la localización.

\section{CONCLUSIONES}

El desarrollo de este ejercicio evaluó cuatro variables que se distribuyen de manera no homogénea en el territorio urbano de Medellín:

i) el espacio público como elemento integrador fundamental para la construcción de ciudad y de ciudadanía, está disperso en parches $y$, aunque tiene algunos elementos de mayores dimensiones que rápidamente resaltan en el mapa, no parece tener una correlación con los costos del suelo y de las viviendas en los barrios de la ciudad. Esto indica que los esfuerzos hechos por la administración municipal y por EPM, para generar nuevos espacios públicos en distintos sitios del territorio, sin privilegiar algunos sectores sobre otros, ha dado como resultado un equilibrio en este indicador. No obstante, toda la ciudad presenta déficit en espacio público efectivo, por lo que es necesario cumplir las metas planteadas en el POT de Medellín e incrementar las áreas disponibles para el esparcimiento, recreación, interacción y convivencia de la población.

ii) las amenazas naturales están presentes en mayor medida en los barrios con rentas por debajo del promedio. No obstante, también se encuentran en algunos de los sectores más exclusivos de la ciudad. En este ejercicio se evaluó la distribución espacial de las amenazas naturales y su correlación con las condiciones socioeconómicas de la población, lo cual no mostró relaciones significativas; sin embargo, cabe anotar que la vulnerabilidad de los habitantes de los sectores más pobres es mayor dadas las técnicas de construcción y las formas de asentamiento.

iii) la calidad del aire es un aspecto que se ha tornado crítico en Medellín y el área metropolitana, especialmente en las épocas de lluvias. Aunque, en promedio, esta variable no muestra una relación significativa con las condiciones socioeconómicas en los barrios, se resalta su concentración en el centro de la ciudad, donde trabajan y por donde transitan diariamente miles de personas. En este sentido, la reducción de los niveles de material particulado y otros contaminantes del aire es prioridad para la gestión ambiental local y compete a todos los ciudadanos de Medellín.

iv) el valor de las viviendas no presentó correlación con las demás variables evaluadas a escala de barrio. No obstante, esta variable muestra una fuerte autocorrelación positiva, que indica que los barrios con las viviendas más costosas están cerca de otros con vivien- 
das de alto valor, mientras que los barrios con viviendas de menor valor están localizados en inmediaciones de sectores con condiciones similares; aunque estos últimos presentan mayores densidades poblacionales.

En el área urbana de Medellín, la distribución espacial de las variables ambientales evaluadas (espacio público, calidad del aire y amenazas

\section{REFERENCIAS}

Acevedo, I. y Velásquez, H. (2008). Algunos conceptos de la econometría espacial y el análisis exploratorio de datos espaciales. Ecos de Economía: A Latin American Journal of Applied Economics, 12(27), 9-34.

Alcaldía de Medellín y BID (2011). Medellín: transformación de una ciudad. doi:10.1017/ CBO97181107415324.004

Alcaldía de Medellín (2014). Acuerdo 48 de 2014 "Por medio del cual se adopta la revisión y ajuste de largo plazo del Plan de Ordenamiento Territorial del Municipio de Medellín y se dictan otras disposiciones complementarias". Medellín: Gaceta Oficial 4267 de 2014.

Alcaldía de Medellín. (2017). Así es Medellín_Medellín Cómo Vamos. Recuperado de https://www.medellincomovamos.org/la-ciudad/ (Recuperado el 1/30/2018).

Alcaldía de Medellín, EDU, Empresa de Desarrollo Urbano, y BID, Banco Interamericano de Desarrollo (2014). Equidad territorial en Medellín. En J. Navarrete Heredia, M. Ángel Bernal y M. Donovan (Eds.) La empresa de desarrollo urbano EDU como motor de la transformación urbana. Medellín: Panamericana Formas e Impresos.

AMVA, Área Metropolitana del Valle de Aburrá (2016). Acuerdo Metropolitano N¹5 "Por el cual se aprueba y se adopta el Protocolo del Plan Operacional para Enfrentar Episodios Críticos de Contaminación Atmosférica en la jurisdicción del Área Metropolitana del Valle de Aburrá". Medellín.

Berroeta, H.; Carvalho, L. y di Masso, A. (2016). Significados del espacio público en contextos de transformación por desastres socionatura- naturales) no se correlaciona con el valor de las viviendas, señalando una aparente equidad territorial tanto en aquellos atributos deseables como en los que no lo son. No obstante, el indicador de autocorrelación espacial (índice I de Moran en este caso) puede quedarse corto como expresión de justicia territorial, ya que una distribución aleatoria o equitativa, no necesariamente es justa.

les. Revista INVI, 31(87), 143-170. doi:10.4067/ invi.v0i0.1042

Boone, C.G.; Buckley, G.L.; Grove Morgan, J. y Sister, C. (2009). Parks and people: An environmental justice inquiry in Baltimore, Maryland. Annals of the Association of American Geographers, 99(4), 767-787. doi:10.1080/00045600903102949

Borja, J. y Muxí, Z. (2000). El espacio público, ciudad y ciudadanía. Barcelona.

Bret, B.; Gervais-Lambony, P. y Hancock, C. (2016). Justicia e injusticias espaciales. Universidad Nacional de Rosario UNR Editora, Rosario, Argentina.

Buzai, G.D. (2016). Geografía aplicada a la solución de problemáticas sociales (pp. 15-29). En Soluciones espaciales a problemas sociales urbanos: aplicaciones de tecnologías de la información geográfica a la planificación y gestión municipal. Sant: Pontificia Universidad Católica de Chile.

Buzai, G.; Lanzelotti, S.; Humacata, L.; Principi, N.; Acuña Suárez, G. y Baxendale, C. (2015). Análisis espacial y evaluación de zonas de potenciales conflictos ambientales, productivos y patrimoniales ante la expansión urbana en la cuenca Del Río Luján (Provincia de Buenos Aires, Argentina). Perspectiva Preliminar (pp. 245-252). En Gestión y ordenamiento territorial. Universidad del Azuay.

Buzai, G. y Villerías Alarcón, I. (2018). Análisis espacial cuantitativo de los determinantes sociales de la salud (DSS) en la cuenca del río Luján (provincia de Buenos Aires, Argentina). Estudios Socioterritoriales, (23), 155-169.

Caballero, J. (2011). Las Avenidas Torrenciales: 
Una Amenaza Potencial en el Valle de Aburrá. Revista Gestión y Ambiente, 14(3), 45-50.

Cárdenas, M.F. y Agudelo, L.C. (2013). La Renta del Suelo Urbano y el Modelo de Expansión Urbana: El Caso de Medellín. Proyección, VII, 74-90.

Celemín, J.P. (2012). Asociación espacial entre fragmentación socioeconómica y ambiental en la ciudad de Mar del Plata, Argentina. Eure, 38(113), 33-51. doi:10.4067/S025071612012000100002

Daza, W. (2008). Espacio público y calidad de vida urbana. Pontificia Universidad Javeriana.

Delgado, G.; Campos, C. y Rentería, P. (2012). Cambio Climático y el Metabolismo Urbano de las Megaurbes Latinoamericanas Climate Change and Urban Metabolism of Latin American Megacities. Habitat Sustentable, 2(1), 2-25.

Gehl, J. (2014). Ciudades para la gente. Buenos Aires: Infinito.

Gómez, F. (2005). Las zonas verdes como factor de calidad de vida en ciudades. Ciudad y Territorio - Estudios Territoriales, 37, 417-436.

Hermelin, M.; Echeverri, A. y Giraldo, J. (2010). Medellín, Medio-Ambiente, Urbanismo y Sociedad. Medellín, Colombia: URBAM - EAFIT Centro de estudios urbanos y ambientales.

Landry, S.M. y Chakraborty, J. (2009). Street trees and equity: Evaluating the spatial distribution of an urban amenity. Environment and Planning $A$, 41(11), 2651-2670. doi:10.1068/a41236

Lucero, P. y Celemín, J. (2008). La calidad de vida de la población en la determinación de la calidad territorial: Un estudio de autocorrelación espacial aplicado a la ciudad de Mar del Plata, Argentina. GeoFocus, (8), 94-114.

Medellín Cómo Vamos. (2018). Informe de Calidad de Vida de Medellín, 2017. Pobreza, desigualdad y demografía. Medellín, Colombia: Alcaldía de Medellín.

Moreno Jiménez, A. (2007). ¿Está equitativamente repartida la contaminación sonora urbana? Una evaluación desde el principio de justicia ambiental en la ciudad de Madrid. Estudios Geográficos, 52(263), 595-626.
Moreno Jiménez, A. (2010). Justicia ambiental. Del concepto a la aplicación en análisis de políticas y planificación territoriales. Scripta Nova: revista electrónica de geografía y ciencias sociales, 14.

Moreno Jiménez, A. (2013). Población y polución atmosférica intraurbana por dióxido de nitrógeno en Madrid: Análisis desde la justicia ambiental basado en sistemas de información geográfica. Cuadernos Geograficos, 52(1), 84-107. Moreno, R. y Vayá, E. (2002). Econometría espacial; nuevas técnicas para el análisis regional: una aplicación a las regiones europeas. Investigaciones Regionales, 1, 83-106.

Ochoa, R. (2011). El espacio público como eje articulador de la calidad de vida en la ciudad de Barranquilla. Jurídicas CUC, 7(1), 139-190.

Ojeda Casares, S.; de Oliveira Neves, G.; Schröder, C. y Hurtado Rodríguez, C. (2008). Equidad territorial en Andalucía. Sevilla: Instituto de Estadística de Andalucía.

OMS, Organización Mundial de la Salud. (2016). Ambient Air Pollution: A global assessment of exposure and burden of disease. Suiza: World Health Organization.

ONU, Hábitat III. (2016). Nueva Agenda Urbana. Quito.

Páramo, P. (2010). El espacio público y la calidad de vida urbana (pp. 15-26). En P. Páramo y E.M. García (Eds.) La dimensión social del espacio público. Bogotá: Universidad Pedagógica Nacional, Universidad Santo Tomás.

Sabatini, F.; Cáceres, G. y Cerda, J. (2001). Segregación residencial en las principales ciudades chilenas: Tendencias de las tres últimas décadas y posibles cursos de acción. Eure, 27(82), 21-42. doi:https://dx.doi.org/10.4067/ S0250-71612001008200002

Sáenz, L. (2016). ¿En qué está el proyecto Parques del Río en Medellín? Arch Daily. Recuperado de https://www.archdaily.co/co/797527/ en-que-esta-el-proyecto-parques-del-rio-enmedellin (consulta 22 de agosto 2016).

Skewes Vodanovic, J.C.; Trujillo Bilbao, F. y Guerra Maldonado, D. (2017). Traer el bosque a sus domicilios. Transformaciones de los mo- 
dos de significar el espacio habitado. Revista INVI, 32(91), 23-64.

Soja, E.W. (2010). Seeking spatial justice. University of Minessota Press.

Subsecretaría de Catastro, A. de M. (2018). Observatorio Inmobiliario de Medellín. Mapa de Ventas. Recuperado de http://catastrooime.blogspot. com/ (consulta 13 de marzo 2018).

Talavera-Dávila, H.V. (2017). Plataforma de cohesión espacial para la inclusión social. Bitacora Urbano Territorial, 27(2), 79-89. doi:10.15446/ bitacora.v27n2.47577

Valencia, N. (2016). La historia de cómo Medellín convirtió sus tanques de agua en verdaderos parques públicos. Arch Daily. Recuperado de https://www.archdaily.co/co/787787/la-historia-de-como-medellin-convirtio-sus-tan- ques-de-agua-en-verdaderos-parques-publicos (consulta 18 de agosto 2018).

Vásquez, A. y Salgado, M. (2009). Desigualdades socioeconómicas y distribución inequitativa de los riesgos ambientales en las comunas de Peñalolén y San Pedro de la Paz. Una perspectiva de justicia ambiental. Revista de Geografía Norte Grande, (43), 95-110. doi:10.4067/ S0718-34022009000200006

Vergara, A.; Gierhake, K.; Jardón, C.; Hernández, J.; Vidal, A. y Carranza, E. (2015). Espacio público en Latinoamérica: de la fragmentación espacial y la segregación social hacia la cohesión territorial. Nuevos retos a viejos problemas. Zentrum für internationale Entwicklungs- und Umweltforschung, 73, 49. Recuperado de http://hdl. handle.net/10419/119871

María Fernanda Cárdenas es PhD en Ingeniería. MSc y Esp. en Planeación Urbano Regional. Ing. Forestal. Su trayectoria profesional ha trasegado entre la consultoría en temas de planeación y ordenación del territorio, y la investigación centrada en ecosistemas estratégicos -como los páramos en Colombia- y los servicios ecosistémicos que ellos proveen, con énfasis en la regulación y el suministro de agua. Actualmente es docente universitaria y ha enfocado su trabajo en temas relacionados con la sostenibilidad ambiental del territorio, estrechamente vinculado con los territorios rurales y su ordenamiento territorial, dentro del grupo de investigación en Dinámicas Urbanas y Territoriales y el grupo EMAT de la Facultad de Arquitectura. Escuela de Planeación Urbano Regional. Facultad de Arquitectura. Universidad Nacional de Colombia. Carrera 65 \#59a-110, Medellín, Antioquia, Colombia, mfcarden@unal.edu.co, ORCID: 0000-0002-1804-6280

Jhon Fredy Escobar es PhD en Administración. MSc en Gestión Tecnológica. Ing. Forestal. Ha trabajado como consultor, docente e investigador en diferentes universidades y centros de educación superior en temas relacionados con la gestión del conocimiento, formulación y gestión de proyectos y sistemas regionales de innovación. Facultad de Ciencias Adminsitrativas. Corporación Universitaria Remington. Calle 51 No. 51-27. Parque Berrio. Medellín, Colombia, jescob1@gmail.com, ORCID: 0000-0002-6826-6222

Katheryn Gutiérrez es Arquitecta. Cursando la maestría en Estudios Urbano Regionales. Ha participado en proyectos de consultoría sobre temas de ordenamiento territorial. En su maestría se centró en estudiar asuntos relacionados con el espacio público urbano con casos de estudio en la ciudad de Medellín. Escuela de Planeación Urbano Regional. Facultad de Arquitectura. Universidad Nacional de Colombia. Carrera 65 \#59a-110, Medellín, Antioquia, Colombia, kgutierrezt@unal.edu.co 